\title{
Effects of ligand substitutions on the rotation rate of indenyl ligands in bis(2- arylindenyl)zirconocenes by NMR lineshape analysis and relaxation in the rotating frame
}

Gregg M. Wilmes, ${ }^{\dagger}$ Marcia B. France, ${ }^{\ddagger}$ Stephen R. Lynch, ${ }^{\dagger}$ and Robert M. Waymouth ${ }^{* \dagger}$

Contribution from the Departments of Chemistry, Stanford University, Stanford, California 94305, and Washington and Lee University, Lexington, Virginia 24450

Supplementary Material

\section{$T_{1 \rho}$ Measurements}

For each spectrometer used for the measurements, the $360^{\circ}$ pulse width $\left(\mathrm{pw}_{360^{\circ}}\right)$ at a range of transmitter powers was determined by obtaining the spectrum of a sharp single peak and varying the pulse width to find that necessary to obtain the $360^{\circ}$ null point. The $360^{\circ}$ pulse width (in seconds) measured for a given transmitter power could then be converted to the field strength, $\omega_{1}$, by equation 1 .

$$
\omega_{1}(\mathrm{rad} / \mathrm{s})=\frac{2 \pi}{\mathrm{pw}_{360^{\circ}}}
$$

The $\mathrm{pw}_{360^{\circ}}$ was measured over a range of transmitter powers, and intermediate values were interpolated by a logarithmic curve. For subsequent data analysis, the field strength obtained at a given transmitter power was obtained from this logarithmic curve. As long as care was taken in retuning the ${ }^{1} \mathrm{H}$ channel of the probe with every temperature change, the pulse-widths, and therefore the field-strengths, were reproducible over time and between samples. The spin-locking field strengths that could be generated by the two instruments used in this study varied somewhat due to different designs of the probes used. The maximum spin-locking field strength that could be generated by the Unity Inova 300 was $140,000 \mathrm{rad} / \mathrm{s}$; that of the Unity Inova 500 was 34,000 rad/s. 
To perform the $T_{1 \mathrm{p}}$ measurement, the sample was brought to the appropriate temperature (calibrated by a $\mathrm{MeOH}$ standard) and a spectrum was obtained with the peak of interest at the center of the spectral window. The spin-lattice relaxation time, $T_{1}$, of the exchanging peak was measured using a standard inversion-recovery pulse sequence generated by the "dot1" macro in the Varian VNMR 6.1 software package. The macro calculated the $T_{1}$ of the resonance along with the error in $T_{1}$ according to the goodness of the fit to an exponential curve. The $T_{1}$ was measured three times for each sample, and the value of $T_{1}$ used was calculated by taking the error-weighted mean of the three measurements.

At each spin-locking field strength, $T_{1 p}(\mathrm{obs})$ was obtained using the pulse sequence: $90^{\circ}{ }_{\mathrm{X}}-(\text { spin-lock })_{\mathrm{Y}}-$ Acquire. A continuous wave spin-lock was used. The phase of the pulse, spin-lock and receiver were incremented $90^{\circ}$ with each scan, with a delay of five times $T_{1}$ between scans. The frequency of the spectrum was centered 5-20 $\mathrm{Hz}$ away from the $\mathrm{Cp}$ peak in order that the intensity could be measured accurately without off-resonance effects due to imperfect receiver balancing. The determination of rates by $T_{1 \mathrm{p}}$ is an on-resonance technique, but is valid in this case as long as the difference between the resonance frequency and the pulse frequency is much lower than the spinlocking field strength. The range of the spin-lock pulse lengths was varied such that $\sim 20$ spectra were obtained in which the intensity of the peak decayed from its maximum to 0 . The time-constant of this decay was obtained by fitting the intensity vs. time data to a first-order exponential decay according to equation 2 with Prism, ${ }^{1}$ where $I_{t}$ is the intensity of a peak with the spin-locking field on for time $t, I_{0}$ is the peak intensity when no spin- 
locking field was used, and $\mathrm{I}_{\infty}$ is the level to which the intensity decayed as $t$ approached infinity.

$$
I_{t}=\left(I_{0}-I_{\infty}\right) \mathrm{e}^{-1 / T_{I \rho} \text { (obs) }}+I_{\infty}
$$

This curve fitting generated a value for $T_{1 \mathrm{p}}(\mathrm{obs})$ and a standard error at the $95 \%$ confidence level. Representative data for (2-(3,5-di-t-Bu-Ph)Ind) $\mathrm{ZrMe}_{2}(\mathbf{2 b})$ at $186 \mathrm{~K}$ are shown in Figure 1.

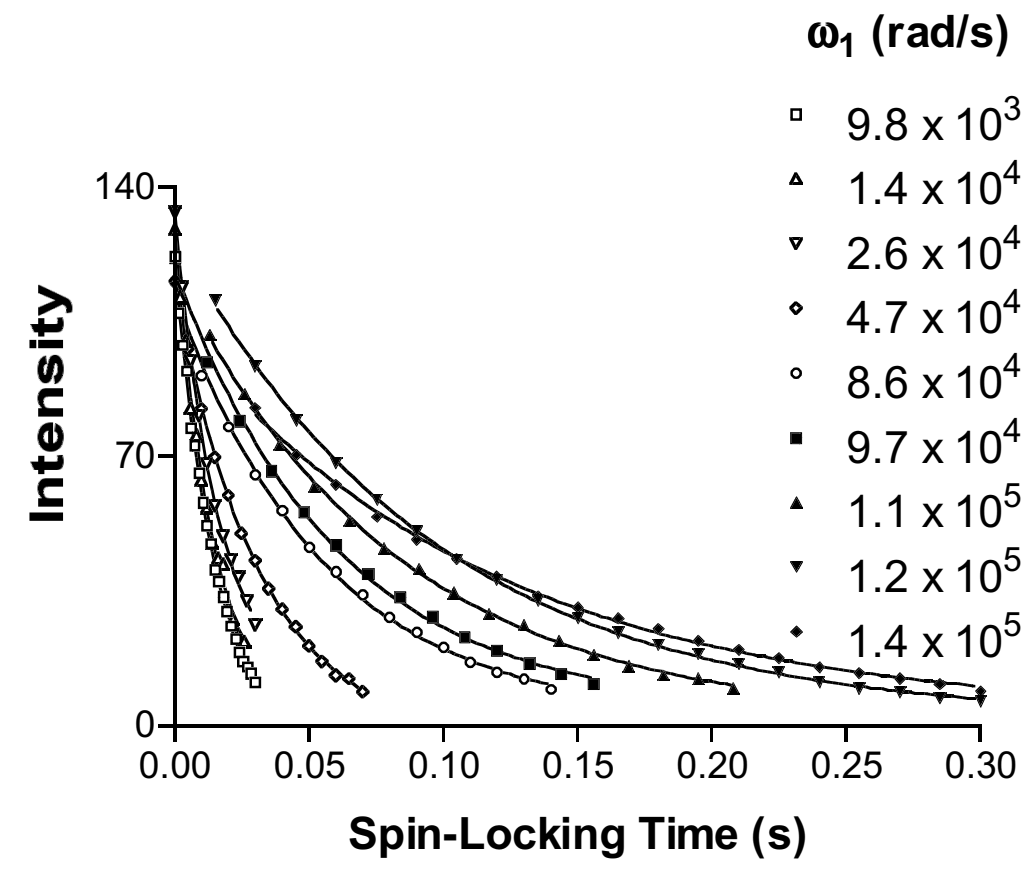

Figure 1. Peak intensity vs. spin-locking time for compound $\mathbf{2 b}$ at $186 \mathrm{~K}$ at differing spin-locking field strengths with best-fit exponential curves.

The best-fit values for $T_{1 \rho}(\mathrm{obs})$ were then converted to $T_{1 \mathrm{p}}(\mathrm{ex})$ using equation 3 , and the error in $T_{1 \rho}(\mathrm{ex})$ was determined by propagation of the errors of $T_{1 \rho}(\mathrm{obs})$ and $T_{1}{ }^{2}$

$$
\frac{1}{T_{1 \rho}(\mathrm{ex})}=\frac{1}{T_{1 \rho}(\mathrm{obs})}-\frac{1}{T_{1}}
$$


The calculated values of $T_{1 \rho}(\mathrm{obs})$ and $T_{1 \rho}(\mathrm{ex})$, with errors, at each spin-locking field strength for $\mathbf{2 b}$ at $186 \mathrm{~K}$ are shown in Table 2.

Table 2. $T_{1 \rho}(\mathrm{obs})$ and $T_{1 \rho}(\mathrm{ex})$ Values for $2 \mathrm{~b}$ at $186 \mathrm{~K}$ Using $T_{1}=0.6027 \pm 0.0017 \mathrm{~s}$

\begin{tabular}{|c|c|c|c|c|c|}
\hline$\omega_{1}(\mathrm{rad} / \mathrm{s})$ & \multicolumn{2}{|c|}{$T_{1 \mathrm{p}}(\mathrm{obs})(\mathrm{s})$} & \multicolumn{3}{|c|}{$T_{1 \rho}(\mathrm{ex})(\mathrm{s})$} \\
\hline $9.8 \times 10^{3}$ & 0.0158 & \pm 0.0006 & 0.0162 & \pm & 0.0006 \\
\hline $1.4 \times 10^{4}$ & 0.0146 & $\pm \quad 0.0011$ & 0.0149 & \pm & 0.0011 \\
\hline $2.6 \times 10^{4}$ & 0.0164 & \pm 0.0007 & 0.0169 & \pm & 0.0007 \\
\hline $4.7 \times 10^{4}$ & 0.0318 & \pm 0.0006 & 0.0336 & \pm & 0.0006 \\
\hline $8.6 \times 10^{4}$ & 0.0550 & \pm 0.0022 & 0.0606 & \pm & 0.0027 \\
\hline $9.7 \times 10^{4}$ & 0.0603 & \pm 0.0029 & 0.0670 & \pm & 0.0035 \\
\hline $1.1 \times 10^{5}$ & 0.0822 & \pm 0.0020 & 0.0952 & \pm & 0.0027 \\
\hline $1.2 \times 10^{5}$ & 0.0959 & \pm 0.0013 & 0.1140 & \pm & 0.0019 \\
\hline $1.4 \times 10^{5}$ & 0.1164 & $\pm \quad 0.0040$ & 0.1443 & \pm & 0.0062 \\
\hline
\end{tabular}

The calculated values of $T_{1 \rho}(\mathrm{ex})$ were then plotted vs. the square of the spinlocking field strength $\left(\omega_{1}^{2}\right)$. A linear least-squares fit, weighted by the inverse square of the error of each value, was generated, and the calculated values for the slope and intercept were used to calculate $\tau_{\mathrm{ex}}(\mathrm{s})$ and $\Delta v(\mathrm{~Hz})$ according to equations 4 and 5.

$$
\begin{gathered}
\tau_{\mathrm{ex}}=\left(\frac{\text { slope }}{\text { intercept }}\right)^{\frac{1}{2}} \\
\Delta v=\left(\frac{\tau_{\mathrm{ex}}}{\pi^{2} \text { slope }}\right)^{\frac{1}{2}}
\end{gathered}
$$

The errors in $\tau_{\mathrm{ex}}$ and $\Delta v$ were calculated by propagation of the standard errors of the slope and intercept at the $95 \%$ confidence level. A representative linear fit for for (2$(3,5 \text {-di-t-Bu-Ph)Ind })_{2} \mathrm{ZrMe}_{2}(\mathbf{2 b})$ at $186 \mathrm{~K}$ is shown in Figure 2. 


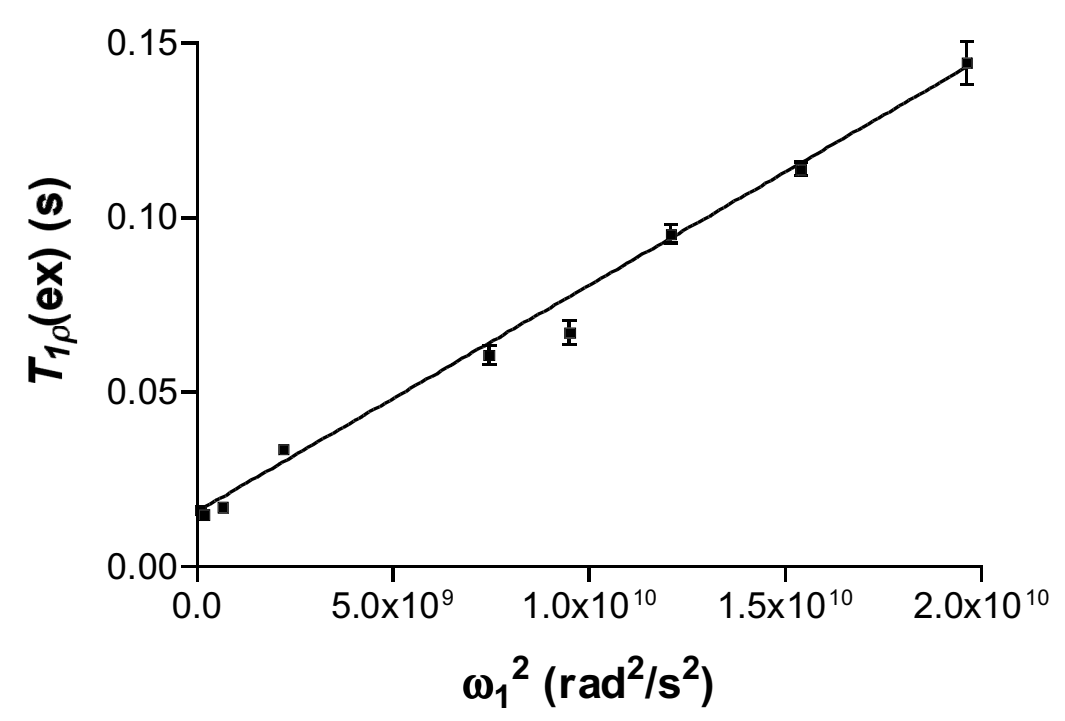

Figure 2. $T_{1 \rho}(\mathrm{ex})$ vs. square of spin-locking field strength $\left(\omega_{1}{ }^{2}\right)$ with weighted best fit line for $\mathbf{2 b}$ at $186 \mathrm{~K}$.

Measurements for the dimethyl compounds were performed 2-4 times at each temperature. The reported rates and peak separations are the weighted averages of the replicates. $^{2}$

Measurements of Dynamic Processes Using NMR Lineshapes All rates of exchange were determined by comparison of experimental spectra to those simulated using the gNMR software package. ${ }^{3}$ Spectra were simulated using the chemical shifts of the exchanging resonances $\left(\delta_{\mathrm{A}}\right.$ and $\left.\delta_{\mathrm{B}}\right)$, the half-height peak width in the absence of exchange (W), the 2-bond $\mathrm{H}-\mathrm{H}$ coupling constant $\left({ }^{2} J_{\mathrm{A}-\mathrm{B}}\right)$, and the first order rate constant of exchange $(\mathrm{k})$. The simulated spectra are quite sensitive to changes in these parameters, and small errors in the measurements (or predictions) of the chemical shifts or peak widths can have large effects on the calculated rate of exchange. Errors in the value of $\mathrm{k}$ were calculated according to methods presented by Sandström, ${ }^{4}$ and are summarized below. 
Chemical Shifts The chemical shifts of both the $\mathrm{Cp}$ and benzyl peaks of (2$\mathrm{PhInd})_{2} \mathrm{ZrBn}_{2}$ (1c) and (2-(3,5-di-t-Bu-Ph)Ind $)_{2} \mathrm{ZrBn}_{2}(\mathbf{2 c})$ were strongly dependent on temperature. To estimate the differences in chemical shifts between temperatures above the coalescence point, the difference between the two peaks was plotted vs. the temperature for all spectra recorded below the coalescence point. A linear least squares fit was made to this plot, and the calculated slope and intercept (along with their errors) were used to estimate the difference of chemical shifts above the coalescence point.

Half-Height Line-Widths At each temperature, the line-width of a reference peak $\left(\mathrm{W}_{\text {ref }}\right)$ was simulated along with the line-width of the exchanging peaks (the reference peak was from the tert-butyl peak in $\mathbf{2 c}$ and from residual 2-phenylindene for 1c). The difference in line-widths $(\Delta \mathrm{W})$ between the reference peak and the peaks of interest in the absence of exchange (W) were measured at sufficiently low temperature that the rate of exchange was essentially zero. At higher temperatures, the half-height peak-width used for the calculation (W) was determined using $\mathrm{W}_{\text {ref }}$ at that temperature and $\Delta \mathrm{W}$ from the nonexchanging case according to equation 6.

$$
\Delta \mathrm{W}=\mathrm{W}-\mathrm{W}_{\text {ref }}
$$

Coupling Constants The two-bond $\mathrm{H}-\mathrm{H}$ coupling constant, ${ }^{2} J_{\mathrm{A}-\mathrm{B}}$, of the diastereotopic benzyl protons was measured at each temperature in the slow exchange regime below temperatures at which line broadening obscured the coupling. The coupling constant used for the exchange simulations at higher temperatures was the average of the lower temperature coupling constants. 
Error Analysis Estimates of the error in rate constant were made differently in the fast and slow exchange regimes. In the slow exchange regime, the rate constant can be estimated using equation 7 , with $\Delta \mathrm{W}_{\mathrm{e}}$, the difference between the observed line-width and the line-width in the absence of exchange.

$$
\mathrm{k}=\pi \Delta \mathrm{W}_{\mathrm{e}}
$$

The error in $\mathrm{k}$, with the addition of a $5 \%$ error for inaccuracies of fitting the simulated peak to the experimental, is given by equation 8 .

$$
\partial \mathrm{k}=\sqrt{\left(\partial \Delta \mathrm{W}_{\mathrm{e}}\right)^{2}+(0.05 \mathrm{k})^{2}}
$$


In the fast exchange regime, above the coalescence temperature, the rate constant of exchange can be estimated by equation 9 , and the error in $\mathrm{k}$ by equation 10 .

$$
\begin{gathered}
\mathrm{k}=\frac{\pi(\delta v)^{2}}{2 \Delta \mathrm{W}_{\mathrm{e}}} \\
\partial \mathrm{k}=\sqrt{\left(\frac{\pi \delta v \partial \delta \mathrm{v}}{\Delta \mathrm{W}_{\mathrm{e}}}\right)^{2}+\left(\frac{\pi(\delta \mathrm{v})^{2} \partial \Delta \mathrm{W}_{\mathrm{e}}}{2 \Delta \mathrm{W}_{\mathrm{e}}^{2}}\right)^{2}+(0.05 \mathrm{k})^{2}}
\end{gathered}
$$

The error in $\delta v$ could be estimated by the errors in the slope and intercept obtained from a linear least squares fit of $\delta v$ to temperature. The values used were $25 \mathrm{~Hz}$ for compound $\mathbf{1 c}$ and $33 \mathrm{~Hz}$ for compound $\mathbf{2 c}$. The error in $\Delta \mathrm{W}_{\mathrm{e}}$ was estimated to be 0.1 $\mathrm{Hz}$ for both compounds.

\section{References:}

(1) Prism, Macintosh version 3.1; GraphPad Software, Inc.: San Diego, 2000 (www.graphpad.com).

(2) Taylor, J. R. An Introduction to Error Analysis; 2nd ed.; University Science Books: Sausalito, California, 1997.

(3) Budzelaar, P. H. M. gNMR, version 4.1; Adept Scientific: Herts, 1999 (www.adeptscience.com).

(4) Sandström, J. Dynamic NMR Spectroscopy; Academic Press: London, 1982. 